\title{
Assessment of Quality of Life and Physical Activity Levels in Greek Breast Cancer Female Patients under Chemotherapy ${ }^{\dagger}$
}

\author{
Argyro Papadopetraki 1, ${ }^{*}$, Helen Karagianni ${ }^{1}$, Anastassios Philippou ${ }^{1}$ and Maria Maridaki ${ }^{2}$ \\ 1 Medical School, National and Kapodistrian University of Athens, 11527 Athens, Greece \\ 2 Faculty of Physical Education and Sport Science, National and Kapodistrian University of Athens, \\ 17237 Athens, Greece \\ * Correspondence: arg.papadopetraki@gmail.com \\ + Presented at the 9th Greek Conference of Biochemistry and Physiology of Exercise, Thessaloniki, Greece, \\ 18-20 October 2019.
}

Published: 2 September 2019

\begin{abstract}
AIM: Research evidence suggests that physical exercise can be part of standard care for breast cancer (BC) patients, contributing to the prevention or reduction of the adverse effects of both the disease and cancer therapies on physiological and quality of life (QoL) parameters. The purpose of this study was to assess QoL and physical activity (PA) levels of BC female patients under chemotherapy and compare them with healthy controls. MATERIAL \& METHOD: 159 females were recruited, $94 \mathrm{BC}$ patients [age, $57.25 \pm 13.59 \mathrm{yrs}$; height, $1.61 \pm 0.05 \mathrm{~m}$; mass, $69.49 \pm$ $12.67 \mathrm{~kg}$; body mass index (BMI), $26.63 \pm 5.36 \mathrm{~kg} / \mathrm{m}^{2}$ ] and 65 healthy women [age, $49.60 \pm 7.80 \mathrm{yrs;}$ height, $1.65 \pm 0.04 \mathrm{~m}$; mass, $69.04 \pm 5.25 \mathrm{~kg}$; BMI, $\left.25.30 \pm 3.95 \mathrm{~kg} / \mathrm{m}^{2}\right]$. Levels of PA were self-estimated using the International Physical Activity Questionnaire. QoL was self-reported by the BC and control groups using the EORTCQLQ-C30 and the SF-36 Health Survey, respectively. RESULTS: BC patients exhibited a total energy expenditure of 2,200 $\pm 1,187$ MET-min/week, of which 1,384 \pm 592 were spent in moderate PAs, $773 \pm 436$ in walking and $43.34 \pm 159$ in vigorous PAs. On the other hand, $49.30 \%$ and $50.70 \%$ of the controls participated in high-intensity and moderate-intensity PAs, respectively. Moreover, controls and $B C$ patients were spending $2.34 \pm 1.05 \mathrm{~h} /$ day and $4.62 \pm 2.58$ $\mathrm{h}$ /day sitting, respectively. BC patients scored their QoL with $63.43 \pm 20.63$ and physical functioning (PF) with $71.48 \pm 23.35$, while their fatigue, pain, and dyspnea scores were $42.28 \pm 20.54,19.44 \pm$ 24.40 , and $25.93 \pm 28.85$, respectively. Negative correlations were found between QoL and fatigue $(p$ $<0.01)$, PF and pain $(p<0.01)$, fatigue $(p<0.01)$ and dyspnea $(p<0.05)$, while a positive correlation was found between QoL and PF $(p<0.01)$. Healthy participants evaluated their QoL as excellent $(10.30 \%)$, very good $(55.17 \%)$, average $(29.30 \%)$, and poor $(3.40 \%)$. CONCLUSION: This study revealed that $\mathrm{BC}$ patients represented moderate to high levels of functioning and QoL and preferred PAs of moderate intensity. Moreover, a positive association was found between PF and QoL in BC patients, while their QoL and functional capacity were compromised by pain, dyspnea, and fatigue. Although the $\mathrm{BC}$ group used to participate in PAs, they accumulated many hours seated, and they were overweight, further supporting the need for these patients to follow the specific exercise recommendations, so as to optimize their benefits from exercise.
\end{abstract}

Keywords: breast cancer; chemotherapy; physical activity; quality of life, QoL 